\title{
Palm Vein Recognition using Scale Invariant Feature Transform with RANSAC Mismatching Removal
}

\author{
Shi Chuan Soh ${ }^{1}$, M. Z. Ibrahim ${ }^{1}$, Marlina Binti Yaknoํㅗ D. J. Mulvaney ${ }^{2}$ \\ ${ }^{1}$ Faculty of Electrical \& Electronic Engineering, University Malaysia Pahang, \\ 26600 Pekan, Pahang, Malaysia \\ sschuan92@gmail.com,zamri@ump.edu.my, marlinayakno@ump.edu.my, \\ ${ }^{2}$ School of Electronic, Electrical and Systems Engineering, Loughborough University, \\ LE11 3TU, United Kingdom \\ d.j.mulvaney@Iboro.ac.uk
}

\begin{abstract}
Palm vein recognition has been gaining increasing interest as a biometric method, although there still remains an issue regarding difficulties in obtaining robust signals. In this paper, the effects of random sample consensus point mismatching removal and the use different wavelengths of illumination on the recognition rate are investigated. The CASIA multi-spectral palm print image database was used to provide input signals and the scale invariant feature transform (SIFT) and random sample consensus (RANSAC) mismatching removal approaches were adopted for vein extraction and point feature matching. The results show that the RANSAC mismatching point removal was able to eliminate outliers while preserving the appropriate SIFT key points and that this led to an improvement in the equal error rate metric, signifying better recognition performance. The palm vein recognition system was found to achieve a better verification rate when infrared illumination in a specific spectral band was used to obtain the palm vein image.
\end{abstract}

Keywords: Vein Recognition, Scale Invariant Feature Transform, Random Sample Consensus

\section{INTRODUCTION}

Advances in the reliability of biometric authentication, such as using fingerprints, voice identification and iris recognition, has led to the introduction of a number of systems that are able to provide personal authentication. Such personal authentication is generally accepted as providing a more secure means of access rather than relying on what individuals know or carry.

Although hand recognition was one of the first forms of biometrics to be used, relatively little research has been published on the use of this approach for authentication purposes. Of the approaches that use the hand, the recognition of its vein pattern is one of the most promising methods, with the potential to characterize images obtained from fingers, the palm-dorsal or the palm area. The use of the vein pattern has 
the advantage that it is harder to forge as the network of blood vessels lies under the skin and so is not immediately visible to the naked eye, unlike many other biometric attributes [2]. Other advantages of using vein patterns for biometrics is that they are believed to be unique to each individual, even identical twins [2], and the pattern does not normally significantly change over an extended period of time. In terms of acquisition, it is possible to design contactless vein pattern acquisition systems; such systems are often more easily accepted by users as any perception of discomfort is minimized and the likelihood of cross contamination is reduced. It is also important to note that the palm is generally the most reliable hand region from which to obtain vein patterns for biometrics, as this region does not normally exhibit hair growth that may affect the quality of the images captured [3].

Based on the current literature relating to palm vein research, vein pattern analysis methods can be classified into four types, namely geometry-based, statistical-based, feature-based and subspace approaches. Geometry-based methods are those that directly use the vein pattern structure information - this approach often has poor discriminatory ability and is highly sensitive to rotation, translation and scales changes [4]. As an example of a geometry-based system, Lee [5] used a two-dimensional Gabor filter with feature matching using Hamming distances for the vein extraction stage. Statistical-based methods calculate characteristics of the vein patterns which are then used in the matching process, but the statistical approach is often sensitive to changes in translation, rotation and scale, making it unsuitable for contactless vein recognition since careful alignment of the capturing equipment would be needed [6]. Feature-based methods include principal components analysis, linear discriminant analysis and independent component analysis, all of which perform dimensionality reduction resulting in a small set of features that can then be used in simplified matching methods requiring a reduced computational overhead. Subspace approaches attempt to reduce the correlation between estimators in an ensemble in a training subset. Subspace methods often use machine learning or artificial intelligence approaches for classification [4].

In this paper, a local invariant feature-based method is proposed that is independent of scale, translational and rotational changes. In the method, key points are extracted as vein pattern features and a Euclidean distance similarity measure is used for authentication. The impacts on the verification rate when performing random sample consensus (RANSAC) mismatching removal and the use of different wavelengths to illuminate the palm are evaluated.

The paper is organized as follows. Section 2 presents the process of the proposed method with subsection image database, preprocessing stage, feature extraction, mismatching point removal, and feature matching. Section 3 gives the experimental results and analysis. Section 4 provides the conclusions. 


\section{PROPOSED METHOD}

\subsection{IMAGE DATABASE}

The CASIA multi-spectral palm vein database was used in this research. The database contains a total of 7200 palm vein images captured from 100 subjects using a multi spectral imaging device. A CCD camera was used to capture images from the hand that was evenly illuminated in turn by light of six different wavelengths, namely $460 \mathrm{~nm}, 630 \mathrm{~nm}, 700 \mathrm{~nm}, 850 \mathrm{~nm}$, and 940nm as well as white light at intervals determined by a purpose-built control circuit. Fig. 1 shows examples of images obtained at the six different illuminating wavelengths. During capture, the subjects were allowed to vary hand postures to a certain extent in order to simulate typical operational usage, while at the same time allowing the investigation of alternative contactless processing approaches that need to be robust to orientation, scale and translation changes.

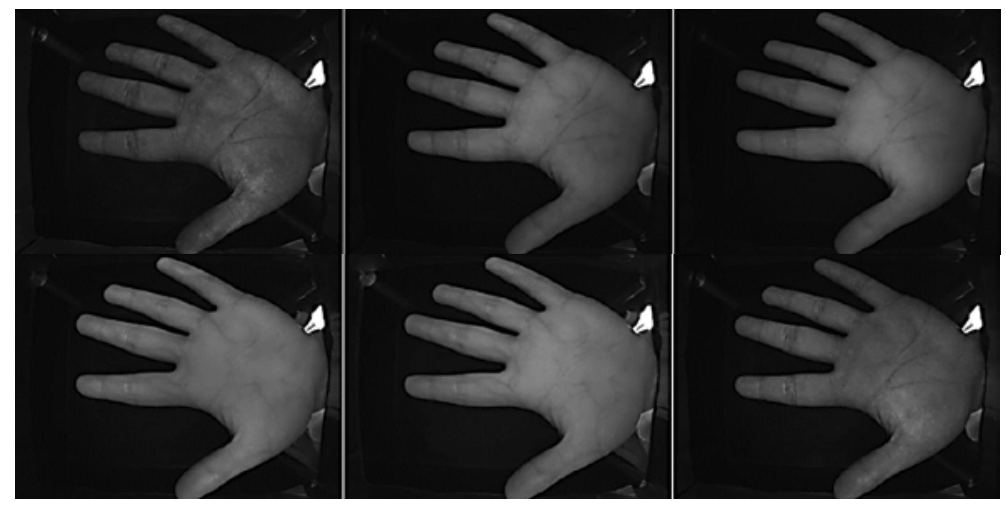

Fig. 1 Images obtained from illumination by the six different wavelengths of light

\subsection{PREPROCESSING}

A low-pass Gaussian filter and Otsu threshold [7] were used to provide an approach with a low computational overhead that was able to reduce the presence in the image of high frequency and background noise. The Otsu technique is able to find a suitable threshold value from a bi-modal gray-level histogram, following which segmentation of the image can be performed according to illumination differences. Morphological filtering of the images was also carried out in order to reduce the presence of a small area of white pixels apparent in a number of the images at a point close to the palm region. Region of interest (ROI) extraction was then applied to remove the background, leaving only the palm region, and the palm images were aligned to a specific orientation to ensure the resulting images are invariant to rotation. Three key points were then generated, namely the valley points between the index and middle finger and between the ring and little finger, as well as the position of the palm point. The images are translated so that the center point of the palm region is the same in all images and the locations of the valley points were then simply scaled accordingly [8]. 
A number of stages of image enhancement were then carried out. Firstly, histogram equalization was used to provide an even distribution of intensities and this was found to make the presence of the veins more apparent to a naked eye observer of the resulting images. In this work, contrast-limited adaptive histogram equalization was applied; this is a popular equalization method that is known to be better than simple histogram equalization at limiting the amplification of noise [9]. The image obtained following ROI extraction was divided into 8x8 pixel blocks and stretched to provide a histogram distribution with a limited and uniform contrast [10]. Finally, 'salt and pepper' noise that often appeared near the vein patterns was removed by using a median filter that is able to conserve the edges in the image.

\subsection{FEATURE EXTRACTION}

The scale invariant feature transform (SIFT) is a feature detection method that is insensitive to rotation, scale and translation changes [11] and was used in the current work to calculate palm vein features that are invariant to scale changes [9].

The implementation of SIFT involves scale space extreme detection, key point localization, orientation assignment and the generation of key point descriptors [12]. The key points are obtained from the local extrema generated by difference of Gaussian operations applied in scale space to a series of resampled versions of the images [13]. Only those key points that exhibit good contrast performance following a repeated scanning of the image over a range of locations and scale are retained. Orientation histograms are then found in order to generate a gradient magnitude for each key point feature. Finally, a 128-dimensional feature vector is formed to specify the image gradient and orientation for each key point.

\subsection{RANDOM SAMPLE CONSENSUS MISMATCHING REMOVAL}

SIFT algorithms use matching between key points in the test and stored images to make authentication decisions. If key points have been incorrectly identified in either of the pairs of an image being compared, then a mismatching is likely to occur and so adversely affect the verification rate. Mismatching is often caused by outliers that result from image processing operations, such as fusion, rotation or resizing. In many cases it is possible to identify when outliers will occur (and so remove them) by estimating the effects that will be caused by applying geometrical transformations to an image.

Random Sample Consensus (RANSAC) is an algorithm to determine the number of inliers that meet the requirements of a pre-defined threshold distance within a certain number of computational iterations [14]. An initial random selection of key point pairs from the SIFT matching results is used to generate a consensus set and its members are assessed to determine whether they fall within the threshold distance. Outliers are removed and the process is repeated iteratively until the consensus set contains the largest number of inliers meeting the threshold criteria is found [15]. 


\subsection{FEATURE MATCHING}

Distance metrics are a popular approach in determining the similarity between two images using the key point features extracted by SIFT. The minimum Euclidean distance can be calculated by using the straight line distance between corresponding pairs of key point coordinates [16]. A linear support vector machine (SVM) can then be used to determine an overall matching score to assess whether authentication can be confirmed. To assess the performance of the vein recognition system, a receiver operating characteristics (ROC) curve can be calculated, the area under which is a useful performance measure, as is a parameter known as the equal error rate (EER) that can also easily be determined.

\section{EXPERIMENTAL RESULT AND ANALYSIS}

Experiments were carried out to evaluate the impacts on the recognition rate of using the RANSAC mismatching point removal and the illumination of the palm using different in wavelengths of light. In these experiments, 20 images from the CASIA database were used to generate primary results. The effectiveness of RANSAC mismatching point removal and the different illumination wavelengths is accessed using the area under the curve (AUC) of the ROC graph as well as the EER value.

Fig. 2(a) and (b) show the matching points found using the SIFT algorithm both before RANSAC mismatching point removal and after point removal, respectively. In Fig. 2(a) it is apparent that a number of mismatching points were present in the image, these are general those lines that cross over a number of the horizontal matches. The outliers that result in these mismatches can easily be identified as lines of significantly longer length than the bulk of the matches found. In order to remove mismatching points, RANSAC removes the outliers and retains only the correctly-matching points [15]. It can be seen in Fig. 2(b) that the mismatching points were removed by RANSAC and only the appropriate matching points were preserved.

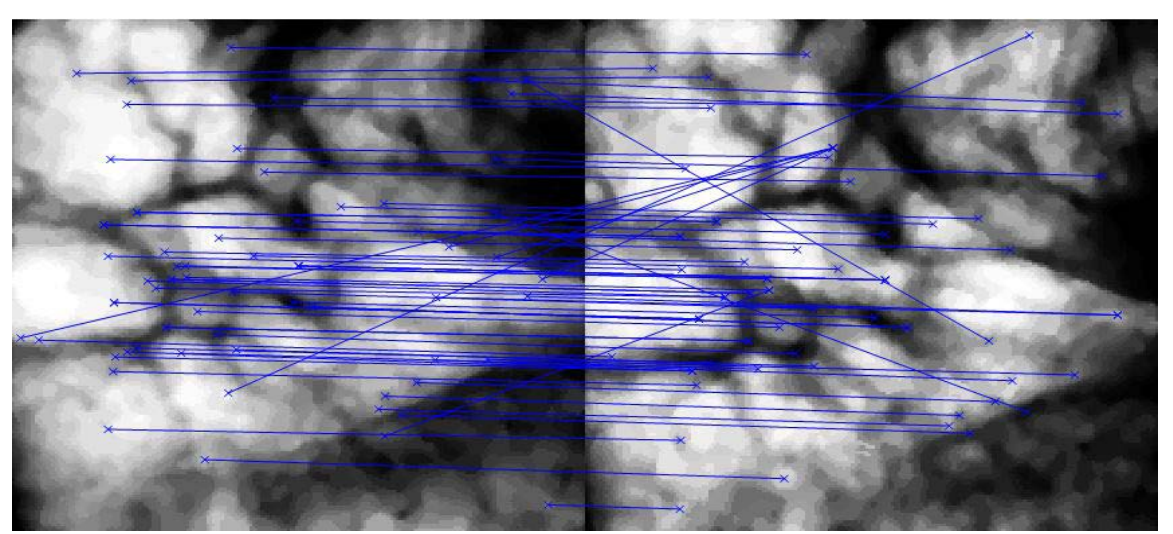

(a) 


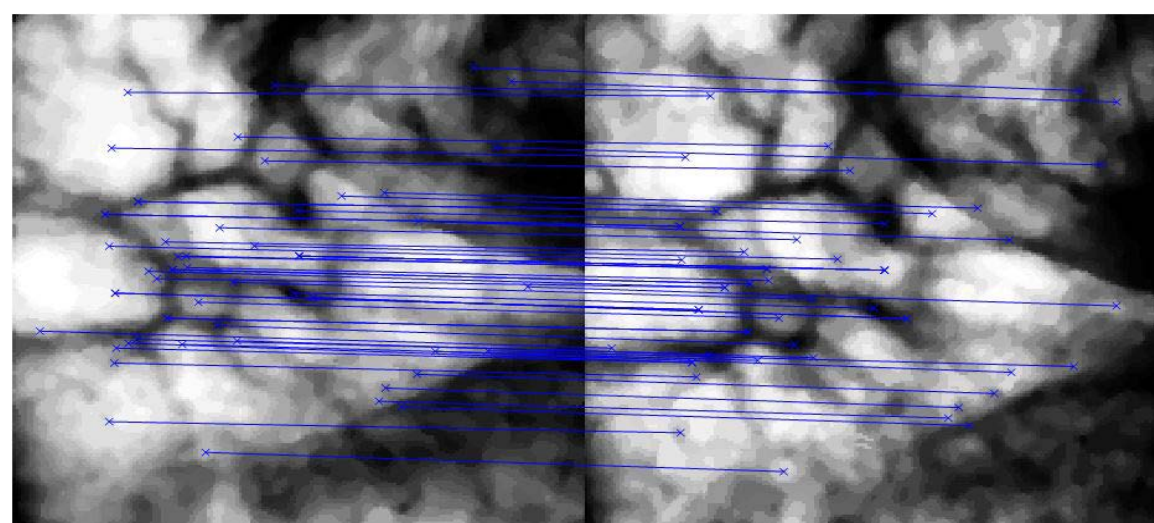

(b)

Fig. 2 SIFT matching (a) before RANSAC mismatching removal, and (b) after RANSAC mismatching removal

Fig. 3(a) and (b) show the ROC curve and EER rate obtained before and after the RANSAC mismatching removal was applied, respectively. For Fig. 3(a) it can be seen that the AUC increases following the RANSAC removal operations and in Fig. 3(b) that the EER rate is reduced by RANSAC, both of which indicate that the verification rate will increase after using mismatching removal. From these two figures, it can be concluded that mismatching removal will have a substantial effect on the successful verification rate of a palm vein authentication system.

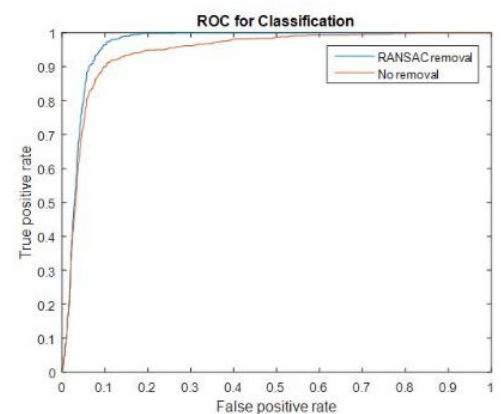

(a)

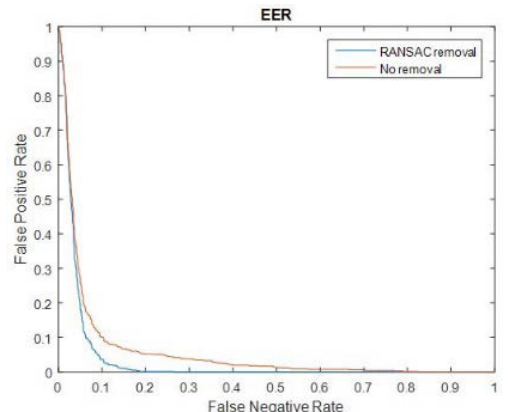

(b)

Fig. 3 Results before and after RANSAC (a) ROC curves (b) EER rate

Fig. 4 shows the effect on the vein verification rate of using three different wavelengths of light for illuminating the palm. It has been found by other researchers that the most suitable band of wavelengths for extracting the palm vein patterns is normally within the infrared region at a range of $700 \mathrm{~nm}$ to $1300 \mathrm{~nm}$ and that the absorption peak is normally located at approximately 970nm [17]. This agrees with the results in Fig. 4(a) and (b), where it can be seen that both the ROC and EER curves show that the verification results are best when using a wavelength of $940 \mathrm{~nm}$ compared to the 
alternative wavelengths of $700 \mathrm{~nm}$ and $850 \mathrm{~nm}$. For the sensor and illumination used for extracting the images in the database, it can be concluded that pattern extraction is best performed at the longer infrared wavelength as more information representing the veins can be extracted.

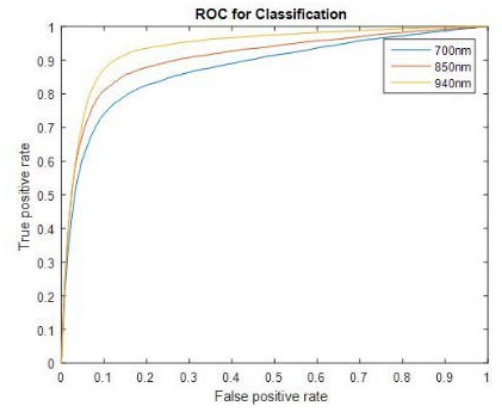

(a)

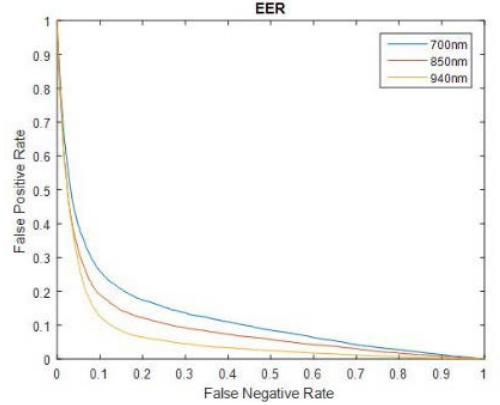

(b)

Fig. 4 Recognition results for three illumination wavelengths (a) ROC curve (b) EER rate

Table 1 shows a summary of the results of the authentication using data obtained both before and after the application of RANSAC and for the three separate illumination wavelengths.

Table 1 Summary of AUC and EER values obtained

\begin{tabular}{lcc}
\hline Method & AUC \% & EER \% \\
\hline SIFT with RANSAC mismatching removal & 96.5 & 7.7 \\
SIFT without mismatching removal & 94.3 & 10.0 \\
SIFT with RANSAC mismatching using 700nm image & 87.7 & 18.2 \\
SIFT with RANSAC mismatching using 850nm image & 90.8 & 14.7 \\
SIFT with RANSAC mismatching using 940nm image & 93.8 & 11.2 \\
\hline
\end{tabular}

\section{CONCLUSIONS}

This paper investigated the effects of palm vein recognition performance of the removal by RANSAC of mismatching points generated by SIFT and of using three different wavelengths of light to illuminate the palm during image acquisition.

Based on the AUC and EER results obtained, the verification rate produced by SIFT was shown to be significantly improved by employing the RANSAC mismatching removal. It is apparent that the removal by RANSAC of the outliers from the consensus set used for matching had a positive impact on the overall verification rate. In addition, it was found that the use of the longer infrared wavelengths for the illumination of the palm allowed the acquisition of images from which it was possible to extract the vein patterns with better quality, thereby giving the potential to simplify the palm vein authentication process. 


\section{ACKNOWLEDGMENTS}

This work is supported by Faulty of Electrical and Electronic Engineering, University Malaysia Pahang under research grant FRGS Grant RDU160108.

\section{References}

1. S.Elnasir andS. M.Shamsuddin, "Palm vein recognition based on 2D-discrete wavelet transform and linear discrimination analysis," Int. J. Adv. Soft Comput. its Appl., vol. 6, no. 3, pp. 43-59, 2014.

2. P.MacGregor and R.Welford, "Veincheck: imaging for security and personnel identification,” Adv. Imaging, vol. 6, no. 7, pp. 52-56, 1991.

3. W.Han and J.Lee, "Expert Systems with Applications Palm vein recognition using adaptive Gabor filter,” Expert Syst. Appl., vol. 39, no. 18, pp. 13225-13234, 2012.

4. W.Kang and Q.Wu, "Contactless palm vein recognition using a mutual foreground-based local binary pattern,” IEEE Trans. Inf. Forensics Secur., vol. 9, no. 11, pp. 1974-1985, 2014.

5. J. C.Lee, “A novel biometric system based on palm vein image,” Pattern Recognit. Lett., vol. 33, no. 12, pp. 1520-1528, 2012.

6. W.Kang, Y.Liu, Q.Wu, and X.Yue, "Contact-Free Palm-Vein Recognition Based on Local Invariant Features,” vol. 9, no. 5, 2014.

7. D.Verma and S.Dubey, "A Survey on Biometric Authentication Techniques using Palm Vein Feature,” vol. 5, no. 8, pp. 2010-2013, 2014.

8. Y.Zhou, A.Kumar, and S.Member, "Human Identi fi cation Using Palm-Vein Images," vol. 6, no. 4, pp. 1259-1274, 2011.

9. M.Pan and W.Kang, "Palm Vein Recognition Based on Three Local Invariant Feature Extraction Algorithms,” pp. 116-124, 2011.

10. H.Zhang, C.Tang, X.Li, A.Wai, andK.Kong, "A Study of Similarity between Genetically Identical Body Vein Patterns."

11. D. G.Lowe, "Object recognition from local scale-invariant features," in Computer vision, 1999. The proceedings of the seventh IEEE international conference on, vol. 2, pp. 11501157, 1999.

12. M. A.Ahmed and A. M.Salem, "Intelligent Techniques for Matching Palm Vein Images 2. Palm Vein Model and Related work,” vol. 39, no. 1, pp. 1-14, 2015.

13. P.-O.Ladoux, C.Rosenberger, and B.Dorizzi, "Palm vein verification system based on SIFT matching," in International Conference on Biometrics, pp. 1290-1298, 2009.

14. Y.Wu, W.Ma, M.Gong, L.Su, L.Jiao, and S.Member, "A Novel Point-Matching Algorithm Based on Fast Sample Consensus for Image Registration,” vol. 12, no. 1, pp. 43-47, 2015.

15. C.Vi and W.G.Vi, "An Integrated Ransac and Graph Based Mismatch Elimination Approach For Wide-Baseline Image Matching,” vol. XL, pp. 23-25, 2015.

16. G.Michael, T.Connie, A.Teoh, T.Connie, and A.Teoh, "A Contactless Biometric System Using Palm Print and Palm Vein Features,” Image (Rochester, N.Y.), 2011.

17. D.Yin andZ.Ding, "Research on Finger Vein Acquisition Based on Wavelength Choice," no. Isci, pp. 2424-2432, 2015. 